\title{
Pix2pix network for fingerprint texture image synthesis
}

\author{
Jader dos Santos Teles Cordeiro, José Hiroki Saito \\ Centro Universitário Campo Limpo Paulista \\ Campo Limpo Paulista, Brasil \\ jaderteles@gmail.com
}

\begin{abstract}
GANs (Generative Adversarial Networks) were proposed to generate realistic synthetic images. In this work, we will discuss the use of GANs as alternative reconstruction of different fingerprint images from the original ones. The samples result in the same person fingerprint but obtained with other textures. Thus, it is intended to contribute to improving the method to increase databases with new samples, incorporating textures, when the quantities are insufficient for any purpose. To verify the similarity of the synthesized images with the original ones, a convolutional Xception network and the RMSE metric are used. The results obtained with fingerprint images of 3 persons, 20 of each finger, and 4 different textures, showed the tradeoff between similarity, recognizability, and the number of epochs of the Pix2pix training.
\end{abstract}

Keywords-GANs, adversarial neural network, generator and discriminator, fingerprints, textures

\section{INTRODUÇÃO}

A identificação individual é utilizada nos mais variados segmentos e a impressão digital é uma das técnicas utilizadas para tal finalidade. Neste sentido, pesquisas em reconstruções realísticas de imagens de impressões digitais com redes neurais, a partir das originais, mantendo a reconhecibilidade, e associando novas texturas, auxiliam no incremento do banco de dados para o desenvolvimento de softwares de reconhecimento.

Diversas pesquisas tem sido realizadas na área de biometria envolvendo reconhecimentos faciais, impressões digitais e detecção de falsificações. Um crescente interesse da comunidade acadêmica pelas pesquisas sobre as vulnerabilidades do reconhecimento facial é relatado por Raghavendra et al. [1].

Uma nova abordagem foi estudada por Li et al. [2], para detectar falsificações, utilizando imagens de raios-x facial em tons de cinza. Jayan e Aneesh [3] atuam no reconhecimento de falsificações com foco em fotografias nas redes sociais.

$\mathrm{Na}$ área de impressões digitais (IDs) são descritas tecnologias empregadas na detecção de simulações construídas para fins de ataques de apresentação. Assim, Rattani et al. [4] descrevem sobre a identificação de materiais fabricados para falsificação de imagens de IDs.

Kulkarni e Sankpal [5] abordam a detecção de IDs na apresentação do dedo vivo avaliando a qualidade da imagem. Com foco na imagem dorsal do dedo, Singh et al. [6] usam câmera de campo de luz (light field camera) no ambiente de ataques de apresentação. Os descritores de textura e redes neurais artificiais são utilizados por Zaghetto et al. [7], na identificação de ataques de apresentação.

No reconhecimento de IDs, Rattani e Ross [8] incluem pré-processamento na distinção entre materiais fabricados. Khutlang e Nelwamondo [9] apresentaram um método de segmentação automática da camada papilar utilizando imagens tomográficas de coerência óptica. O contorno papilar representa a impressão digital interna, que não sofre problemas externos da pele. Pala e Bhanu [10] utilizam aprendizado profundo na distinção entre IDs reais e falsas.

Chugh et al. [11] descrevem que os ataques de falsificação de IDs são crescentes, podendo ser realizados com uma variedade de processos e materiais. Apresenta como exemplo, uma transferência de características de textura entre imagens de IDs, sintetizando com material desconhecido novas IDs.

Wang et al. [12] evidenciam melhorias com o aprendizado profundo no reconhecimento da impressão palmar. Avanços importantes foram apresentados por Tariq et al. [13] para classificadores baseados em redes neurais e na detecção de faces humanas. Tan et al. [14] exploram a identificação de faces falsas geradas por GANs.

GANs (Generative Adversarial Networks) são estruturas de redes neurais projetada inicialmente por Goodfellow et al. [15], integradas por duas redes que disputam uma com a outra, a rede geradora e a rede discriminadora. A rede Pix2pix de Isola et al. [16] tem características semelhantes, mas possui um fator de condição, que serve de critério para se obter novas amostras, e é conhecida como cGAN (GAN condicional). Nos ensaios experimentais realizados e também em pesquisas relatadas na literatura, constata-se a versatilidade das cGANs na geração de imagens sintéticas.

As técnicas de reconstrução de imagens utilizando cGANs, permitem reconstruir imagens de IDs realísticas a partir de IDs originais, podendo auxiliar no incremento de bancos de dados, aumentando a quantidade de amostras de IDs.

Neste trabalho, cGAN Pix2pix é utilizada para gerar imagens sintéticas, numa metodologia denominada SIMTEX (Simulação de textura). As imagens de IDs originais são utilizadas para a obtenção de novas IDs, com pequenas 
variações, porém, mantendo a reconhecibilidade, incluindo texturas variadas. A pesquisa tem como objetivo contribuir na ampliação das amostras num banco de dados, com quantidades insuficientes para finalidades específicas de pesquisa.

O restante do texto está organizado da seguinte forma. $\mathrm{Na}$ Seção II, descrevemos a rede Pix2pix utilizada para a geração de imagens sintéticas, a rede Xception que é utilizada para o reconhecimento das IDs e cálculo da similaridade, os bancos de dados de IDs e texturas utilizados, e a metodologia; na Seção III são descritos os experimentos realizados; e na Seção IV, as conclusões finais.

\section{MATERIAIS E MÉTODOS}

Neste trabalho, utiliza-se duas estruturas de redes neurais. A Pix2pix [16] é aplicada para abordar a associação de novas texturas em IDs, na metodologia SIMTEX, com a finalidade de gerar imagens sintéticas com qualidade realística a partir das reais e a rede Xception (Extreme Exception), proposta por Chollet [17], para o reconhecimento das impressões digitais e também para estimar a proporção de similaridade entre as imagens de IDs.

\section{A. Pix2pix}

Pix2pix surgiu para evidenciar uma aplicação de uso geral das GANs e possui um gerador baseado na arquitetura U-Net, uma rede convolucional proposta por Ronneberger et al. [18] e um discriminador (classificador convolucional) que utiliza uma arquitetura PatchGAN, em que a discriminação ocorre por frações de imagens (patch).

Na rede Pix2pix, o Gerador G é responsável pela geração da imagem y, a partir de um arranjo de ruído $\mathrm{z}$ e da imagem condicional $\mathrm{x}$, ou seja, realiza o mapeamento $\mathrm{G}:\{\mathrm{x}, \mathrm{z}\} \rightarrow \mathrm{y}$. O Discriminador $\mathrm{D}$ é responsável pela classificação da imagem sintetizada $y$, utilizando um conjunto de amostras de imagens $\mathrm{x}$ e determinando a probabilidade de y ser real ou falsa, fazendo portanto o mapeamento $\mathrm{D}:\{\mathrm{x}, \mathrm{y}\} \rightarrow[0,1]$.

Desse modo, pode-se entender Pix2pix como um jogo de minimizador de $\mathrm{G}$ e maximizador de $\mathrm{D}$, que acontece em etapas alternadas, com ajustes nos parâmetros, buscando a otimização, até um resultado satisfatório.

A equação 1 da função objetivo da rede Pix2pix mostra que o gerador $\mathrm{G}$ tenta minimizar a diferença entre a imagem gerada y a partir do vetor de ruído $\mathrm{z}$ e a imagem $\mathrm{x}$; e o discriminador $\mathrm{D}$ tenta maximizar essa diferença.

$$
\begin{array}{r}
\mathrm{L}_{c G A N}(G, D)=\mathrm{E}_{\mathrm{x}, \mathrm{y}}[\log D(\mathrm{x}, \mathrm{y})]+ \\
\mathrm{E}_{\mathrm{x}, \mathrm{z}}[\log (1-D(\mathrm{x}, \mathrm{G}(\mathrm{x}, \mathrm{z})))]
\end{array}
$$

$\mathrm{Na}$ equação 2, temos a minimização desse objetivo feita por $\mathrm{G}$, contra um adversário D que tenta maximizá-lo.

$$
G^{*}=\arg \min _{\mathrm{G}} \max _{\mathrm{D}} \mathcal{L}_{c G A N}(G, D)=\lambda \mathcal{L}_{\mathrm{L} 1}(G)
$$

A função de perda procura mensurar a diferença entre a saída prevista e a saída real desejada pelo treinamento. A cada iteração, a rede $\mathrm{G}$ aprimora os resultados, aproximandoos cada vez mais das reais, e a rede D classifica a amostra gerada como real ou falsa, indicando a necessidade ou não do aperfeiçoamento da rede G.

\section{1) Arquitetura do gerador $G$}

A arquitetura do módulo gerador tem sua estrutura baseada na U-Net [18] e possui semelhanças em relação à proposta de Radford et al. [19], tendo uma forma convolucional convencional na primeira parte, onde as dimensões das matrizes de atributos das camadas sofrem contração. Porém, existe uma segunda parte, de expansão, em que a estrutura evolui aumentando as dimensões das matrizes, até atingir a matriz final com as mesmas dimensões da imagem de entrada, que corresponde à imagem gerada.

Nas camadas de contração, encontram-se uma sequência de filtros de convolução $3 \times 3$, seguida de funções de ativação ReLU (Rectified Linear Unit) [20] e operações de maxpooling $2 \times 2$ com passo 2 . Na camada de expansão, cada etapa consiste na amostragem do mapa de características seguido por uma convolução transposta $2 \times 2$, up-convolution, uma concatenação com as camadas de contração correspondentes e duas convoluções $3 \times 3$, cada uma seguida de uma ReLU. Na última camada, há uma convolução $1 \times 1$ que mapeia os vetores de atributos de 64 elementos para o número desejado de classes.

\section{2) Arquitetura do discriminador $G$}

Para a rede discriminadora é utilizada a arquitetura PatchGAN que faz a discriminação na escala de frações (patch) da imagem y e verifica a semelhança dessas frações com as frações correspondentes das imagens reais $x$. Segundo Isola et al. [16], ao utilizar frações grandes, diminui o tempo de processamento e para otimizar ainda mais esse tempo é implementado o modelo proposto por Ioffe e Szegedy [21], um minilote de treinamento (minibatch), para conseguir altas taxas de aprendizado.

Uma abordagem em rede profunda de Li e Wand [22] com o discriminador PatchGAN sobre frações de imagens, permite que a aprendizagem seja direcionada para o mesmo contexto com diferentes descrições. O discriminador é treinado para distinguir as frações de imagens sintetizadas amostradas com as da imagem alvo, pontuando cada fração da imagem considerada como real com o valor $\mathrm{s}=1$, e $\mathrm{s}=0$, caso contrário.

\section{B. Xception}

A rede Xception [17] é uma rede neural convolucional, utilizada neste trabalho, para o reconhecimento das IDs e para a estimativa de similaridade entre as imagens de IDs geradas utilizando a metodologia SIMTEX e as imagens originais. Possui implementação de conexões residuais que resulta em robustez sem perder a precisão, e tem a possibilidade de mensurar a similaridade entre imagens em uma escala de 0 até 1 .

As conexões residuais profundas foram propostas por $\mathrm{He}$ et al. [23] para evitar a degradação durante o treinamento, com a perda de precisão. Uma versão modificada, mas mantendo os benefícios da abordagem original, foi integrada na arquitetura da rede Xception.

\section{Banco de dados de textura}

A fim de se obter informações de texturas para o SIMTEX, utiliza-se neste trabalho um banco de dados com texturas descritíveis [24], denominado DTD (Describable Textures Dataset), composto por 5.640 imagens de objetos 
diversos, organizado com 47 categorias (classes) de acordo com a percepção humana, com 120 imagens por categoria, nos tamanhos de 300x300 e 640x640 pixels.

Nos experimentos realizados, as classes blotchy, bumpy, crosshatched e fibrous, do banco DTD, foram selecionadas devido a existência de nervuras semelhantes às existentes em IDs e redimensionamos para uma compatibilidade com a rede, as imagens para $256 \times 256$ pixels com o software GIMP (GNU Image Manipulation Program).

\section{Banco de dados de IDs}

Neste trabalho, foi desenvolvido um banco de dados de IDs próprio (Banco de Dados Próprio - BDP) composto por vinte imagens originais para cada dedo da mão esquerda de 3 indivíduos, que resultaram em 300 imagens originais, divididas em 15 classes de dedos individuais. Cada imagem foi coletada utilizando um mesmo protocolo, a partir do posicionamento do dedo no dispositivo de captura, de forma natural, sem a indicação do ângulo de inclinação, mantendo a expontaneidade dos indivíduos. A aquisição das imagens originais foi em 3096x3096 pixels.

\section{E. Pré-processamento e SIMTEX}

$\mathrm{Na}$ fase de pré-processamento, foi aplicado o método ROI (Region Of Interest) para imagens de 3096x3096 pixels do BDP, definindo uma região de interesse de 1500x1500 pixels, correspondente a uma área específica que abrange a parte do dedo que possui a impressão digital. Em seguida, essas imagens foram redimensionadas para o tamanho de 256x256 pixels com o software GIMP, mantendo a compatibilidade com a rede Pix2pix utilizada posteriormente.

Uma equalização adaptativa de histograma limitada pelo contraste foi utilizada para melhorar o reconhecimento de impressões digitais, incorporando o método CLAHE (Contrast-Limited Adaptive Histogram Equalization)[25] [26], para ajustar o nível de cinza, onde o contraste é limitado.

A metodologia SIMTEX (Simulação de Textura), associa uma imagem de textura do banco DTD, como a entrada $\mathrm{z}$ da rede Pix2pix, além de uma imagem $x$, que é uma ID original do banco de dados BDP, para a obtenção da imagem y pela rede G. A Fig.1 ilustra um diagrama da metodologia SIMTEX para a geração de IDs acrescidas de texturas.

A metodologia SIMTEX é iniciada pelas entradas da imagem de ID original $(\mathrm{x})$, mostrada no canto direito superior do diagrama, e a imagem de textura $(\mathrm{z})$, no canto esquerdo superior. As duas imagens passam por um préprocessamento, seguido da equalização de histograma (CLAHE), antes da aplicação da rede Pix2pix para a geração de IDs com textura, na saída (y), mostrada na parte inferior do diagrama.

Denominamos "transferência de aprendizagem" a associação da textura às IDs originais, considerando-se que a rede Pix2pix aplica o modelo aprendido da textura (z), para a imagem da ID original (x). Como resultados teremos sínteses de IDs com textura. Para a obtenção de resultados que conservem as originalidades das IDs, mantendo a sua reconhecibilidade, verifica-se a necessidade de controlar o treinamento da rede pela limitação do número de épocas de treinamento.

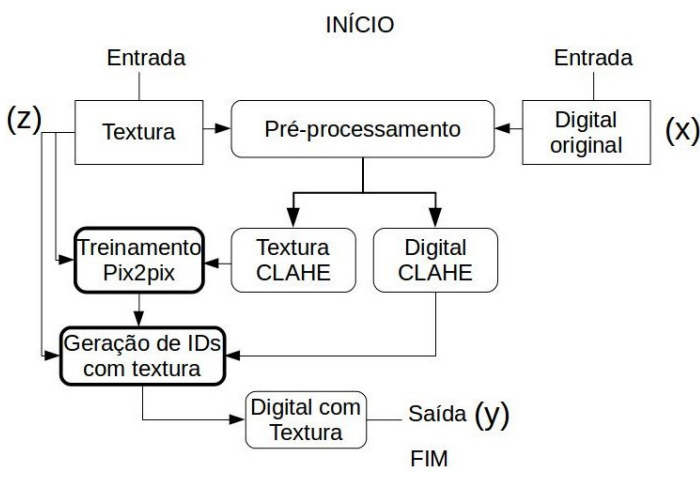

Fig. 1. Estrutura da metodologia SIMTEX para geração de IDs com textura pela rede Pix2pix.

Para verificar a diferença estrutural entre imagens de IDs foi utilizada nos experimentos o cálculo da distância pela raiz quadrada do erro médio quadrático, RMSE (Root Mean Square Error), também conhecida por distância Euclidiana ou Norma L2 [27] [28], com os valores de pixels em nível de cinza no intervalo $[0,255]$. O resultado foi normalizado dividindo o RMSE por 255.

\section{RESUlTAdOS E DISCUSSÕES}

Nesta seção são descritos alguns dos experimentos e os resultados obtidos. No Experimento 1, foi utilizada a rede Pix2pix no método SIMTEX para incorporarmos texturas, ou seja, possibilitar uma combinação de imagens com texturas na constituição de IDs. Já no Experimento 2, as imagens sintéticas geradas pela rede Pix2pix passam pelo reconhecimento, utilizando a rede Xception, e no Experimento 3, são calculadas as similaridades entre as imagens originais e as geradas pelo SIMTEX, utilizando RMSE e a rede Xception.

\section{A. Experimento 1 - Geração de IDs com textura}

A Fig. 2 mostra os resultados visuais da síntese de IDs com o SIMTEX, sendo (a) imagem de ID original, (b) texturas do banco DTD, blotchy, bumpy, crosshatched $e$ fibrous, da esquerda para a direita, e (c) IDs sintetizadas, utilizando as texturas do item anterior, nas respectivas colunas, e variando as épocas de treinamento, de 6, 200, 300 e 400, de cima para baixo.

Durante o experimento, para obtermos amostras reconhecíveis com textura, necessitamos diversificar a quantidade de épocas de treinamento da rede Pix2pix, verificando os resultados de reconhecimento. Neste contexto, apresentaremos no Experimento 2 as diferenças dos resultados com 6, 200, 300 e 400 épocas geradas na rede Pix2pix. A cada nova textura, nos procedimentos temos resultados diferentes de reconhecibilidade, porém, é possível verificar na média, os possíveis números de épocas de treinamento para o SIMTEX. 
(a)
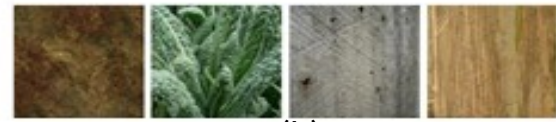

(b)
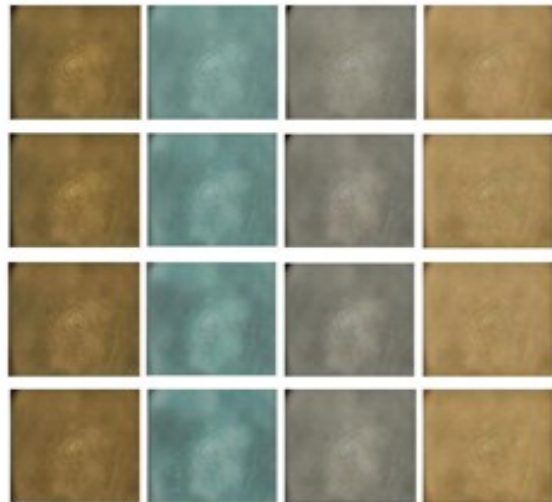

(c)

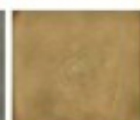

Fig. 2. (a) ID original; (b) texturas blotchy, bump, crosshatched e fibrous (esquerda para a direita); (c) IDs sintetizadas pelo SIMTEX, utilizando as texturas respectivas do item anterior, em 6, 200, 300 e 400 épocas (de cima para baixo), respectivamente.

\section{B. Experimento 2 - Reconhecimento com Xception}

Para verificar a reconhecibilidade das amostras de ID sintetizadas pelo SIMTEX, realizou-se a aplicação da rede Xception para o reconhecimento das IDs obtidas com números diferentes de épocas de treinamento do Pix2pix. Assim, vinte IDs sintetizadas de cada dedo de 3 pessoas com quatro texturas específicas, foram consideradas.

Nos procedimentos para estimar o reconhecimento, foi convencionado que as IDs sintetizadas com textura deveriam alcançar índices de 0,80 ou superiores, na escala de 0 a 1 , para todas as 15 classes (dedos individuais), para serem consideradas reconhecíveis. Quando as vinte amostras de impressões digitais sintetizadas de cada classe são reconhecidas, tem-se $100 \%$ de reconhecimento, ou índice igual a 1 .

Na Fig. 3 é mostrado o resultado dos testes de reconhecimento, de IDs com texturas sendo: (a) blotchy, (b) bumpy, (c) crosshatched e (d) fibrous. Os testes de reconhecimento foram realizados, treinando a rede Xception com as amostras originais das impressões digitais dos 5 dedos da mão esquerda de 3 indivíduos, contido no banco de dados BDP e aplicando os testes de reconhecimento para as amostras geradas pelo SIMTEX.

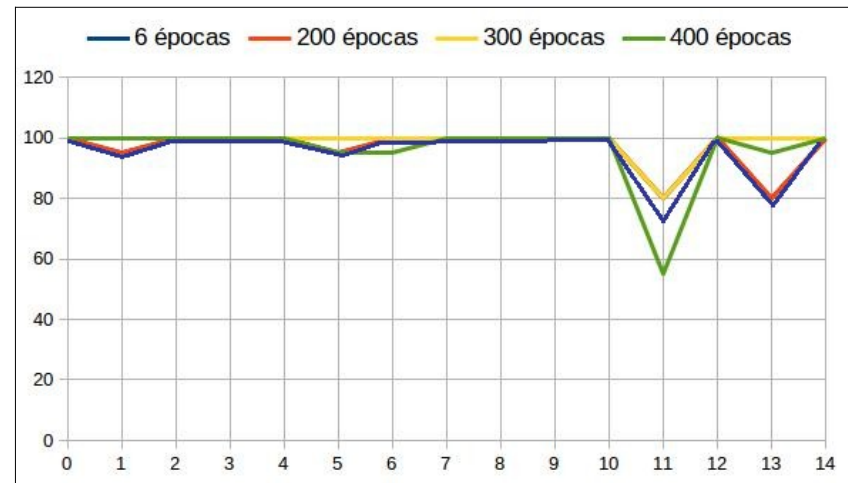

(a)

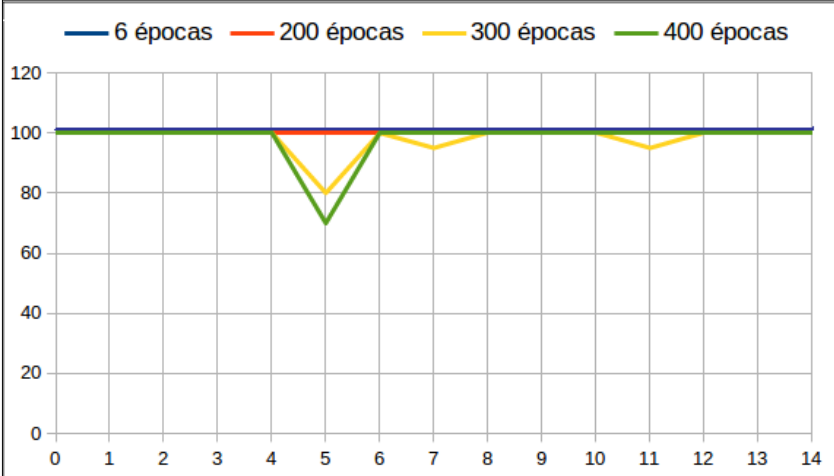

(b)

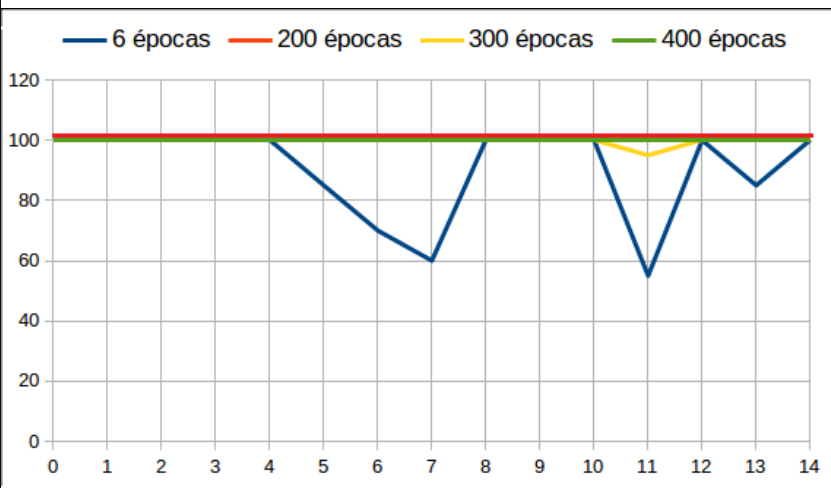

(c)

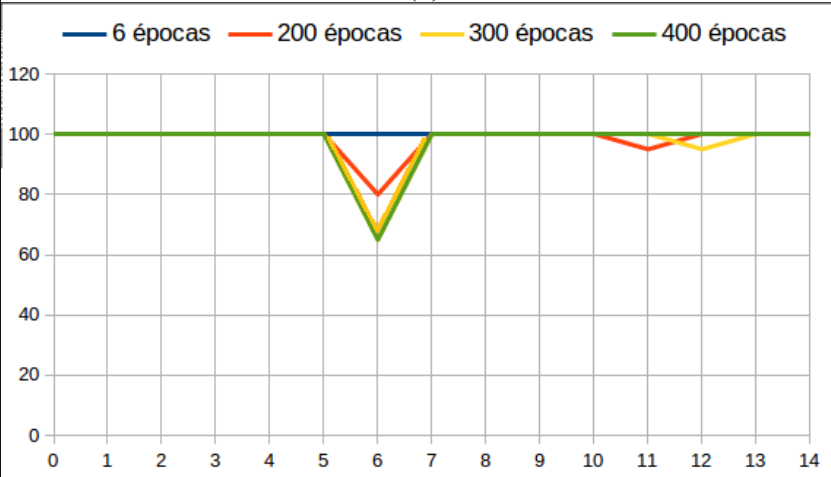

(d)

Fig. 3. (a) blotchy, (b) bumpy, (c) crosshatched e (d) fibrous Similaridade na textura em IDs m 6, 200, 300 e 400 épocas 
A coordenada horizontal no eixo $\mathrm{x}$, corresponde às 15 classes de IDs, cada classe correspondente a um dedo. A coordenada vertical no eixo $\mathrm{y}$, corresponde às porcentagens de reconhecimento. Para cada quantidade de épocas de treinamento aplicadas no Pix2pix, é mostrado um gráfico de porcentagem de reconhecimento, sendo cor azul (6 épocas), vermelha (200 épocas), amarela (300 épocas) e verde (400 épocas).

Os valores obtidos de reconhecimento foram significativamente elevados na maioria das classes, com exceção das classes 5, 6, 7 e 11 , cuja porcentagem de reconhecimento foi abaixo de $80 \%$, para alguns casos descritos a seguir.

Na Fig. 3(a) o reconhecimento foi de $75 \%$ para 6 épocas, e 55\% para 400 épocas, ambos na classe 11 . Na Fig. 3(b) o reconhecimento foi de $70 \%$ na classe 5 em 400 épocas. Na Fig. 3(c) o reconhecimento foi de $70 \%, 60 \%$ e $55 \%$, para as classes 6,7 e 11, respectivamente, em 6 épocas. Já na Fig. 3(d) o reconhecimento foi de $65 \%$ para 300 e 400 épocas, na classe 6. A Fig. 4 ilustra os casos citados num gráfico onde a coordenada horizontal representa o número de épocas e a vertical, o número de classes em que a porcentagem foi abaixo de $80 \%$. Os resultados de reconhecimento mostram uma tendência de que, quando o número de épocas é insuficiente, no caso, 6 épocas, a quantidade de erros é alta. Supomos que a quantidade de ciclos onde os pesos são atualizados, não foi razoável para uma melhor constituição da imagem.

Esse número reduz para um número de épocas do treinamento suficiente (200 épocas), porém os erros voltam a aumentar com quantidade de épocas maiores. O uso de diferentes épocas nos experimentos foi considerado para a verificação empírica do efeito da quantidade dessas épocas. Assim, verificamos o aumento dos erros com 300 e 400 épocas. No mesmo sentido incluímos 6 épocas, na intenção de verificar a possibilidade de gerar imagens razoáveis, mesmo com número reduzido de épocas.

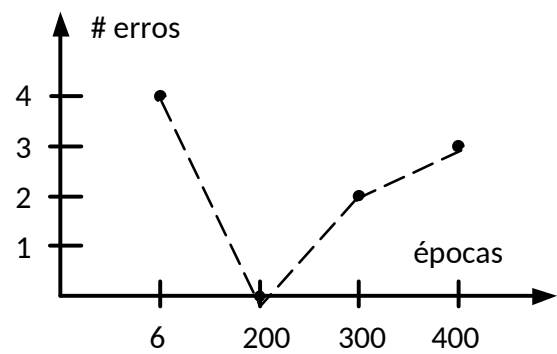

Fig. 4. Quantidade de erros de reconhecimento para diferentes números de épocas de treinamento.

Observa-se, também, que as especificidades de cada classe de textura, associada às características das IDs, não proporcionam resultados idênticos. Por isso, diferenças podem ser vistas nos gráficos para as diversas texturas da Fig.3.

\section{Experimento 3 - Medidas de similaridade}

Conforme descrito na Seção III.E, além da rede Xception que calcula a similaridade, foi utilizada a métrica RMSE, distância Euclidiana ou Norma L2. Foi preciso implementar a normalização dos níveis de intensidade da escala de cinza, e o cálculo da similaridade como a fração complementar da distância normalizada. As Tabelas 1, 2, 3 e 4 apresentam a média e o desvio padrão da similaridade na métrica RMSE na coluna da esquerda, e Xception na coluna da direita, entre as IDs originais e as obtidas pelo SIMTEX para as texturas blotch, bumpy, crosshatched e fibrous, respectivamente. Nota-se que quanto mais próximo de 1, maior é a similaridade entre as amostras de IDs. Os maiores valores de similaridade estão destacados em negrito, e os menores valores, em vermelho. Nota-se que os valores de similaridade são muito próximos para todas as quantidades de épocas de treinamento, e provavelmente por esta razão, os valores mínimos e máximos destacados não coincidem para ambas as métricas. Nota-se também que as medidas de similaridade não refletem na reconhecibilidade das IDs.

\section{CONCLUSÕES E TRABALHOS FUTUROS}

Descrevemos neste artigo uma abordagem experimental denominada SIMTEX, para a obtenção de novas amostras de IDs a partir das amostras de IDs originais, acrescentando texturas, com a intenção de aumentar o repertório de amostras em bancos de dados de IDs. Os resultados mostram que a escolha das texturas é importante para manter a reconhecibilidade das amostras obtidas, além da quantidade de épocas de treinamento. Uma tendência verificada é que um certo número de épocas de treinamento são necessárias para se chegar a um bom índice de reconhecimento, porém, a partir disso, quanto maior a quantidade de épocas de treinamento, a reconhecibilidade fica reduzida. Nota-se que a similaridade entre as amostras originais e as geradas, apesar de altas, a reconhecibilidade das amostras geradas pode ser baixa. Como trabalho futuro, um estudo poderia ser realizado, medindo a similaridade das amostras, cujas IDs não foram reconhecidas pelo Xception. Isso poderia explicitar melhor se existe uma correspondência entre a similaridade e a reconhecibilidade, uma vez que, neste trabalho, os experimentos de similaridade mostram a média das similaridades e não a similaridade específica.

Tabela 1. Impressões digitais com textura blotch

\begin{tabular}{|c|c|c|c|c|}
\hline \multirow[b]{3}{*}{ Épocas } & \multicolumn{4}{|c|}{ Resultados métricos com textura blotch } \\
\hline & \multicolumn{2}{|c|}{ RMSE } & \multicolumn{2}{|c|}{ Xception } \\
\hline & Média & Desvio & Média & Desvio \\
\hline 6 & 0,95918 & 0,00064 & 0,97628 & 0,04651 \\
\hline 200 & 0,95918 & 0,00064 & 0,97626 & 0,04650 \\
\hline 300 & 0,95915 & 0,00057 & 0,99003 & 0,02677 \\
\hline 400 & 0,95893 & 0,00062 & 0,96860 & 0,08590 \\
\hline
\end{tabular}

Tabela 2. Impressões digitais com textura bumpy

\begin{tabular}{|l|l|l|l|c|}
\hline \multirow{2}{*}{} & \multicolumn{3}{|c|}{ Resultados métricos com textura bumpy } \\
\cline { 2 - 5 } Épocas & Média & Desvio & Média & Desvio \\
\hline 6 & $\mathbf{0 , 9 7 1 5 7}$ & 0,00446 & $\mathbf{0 , 9 9 9 6 9}$ & 0,00070 \\
\hline 200 & 0,96980 & 0,00225 & 0,99828 & 0,00419 \\
\hline 300 & 0,96733 & 0,00264 & 0,97830 & 0,04947 \\
\hline 400 & 0,96541 & 0,00201 & 0,98325 & 0,05052 \\
\hline
\end{tabular}


Tabela 3. Impressões digitais com textura crosshatched

\begin{tabular}{|l|l|l|l|l|}
\hline \multirow{2}{*}{} & \multicolumn{2}{|c|}{ Resultados métricos com textura crosshatched } \\
\cline { 2 - 5 } Épocas & Média & Desvio & Média & Desvio \\
\hline 6 & $\mathbf{0 , 9 7 1 3 0}$ & 0,00400 & 0,94098 & 0,09315 \\
\hline 200 & 0,96532 & 0,00315 & 0,99978 & 0,00059 \\
\hline 300 & 0,96454 & 0,00252 & 0,99915 & 0,00314 \\
\hline 400 & 0,96790 & 0,00419 & $\mathbf{0 , 9 9 9 9 2}$ & 0,00025 \\
\hline
\end{tabular}

Tabela 4. Impressões digitais com textura fibrous

\begin{tabular}{|l|l|l|l|l|}
\hline \multirow{2}{*}{} & \multicolumn{3}{|c|}{ Resultados métricos com textura fibrous } \\
\cline { 2 - 5 } & \multicolumn{3}{|c|}{ RMSE } & \multicolumn{2}{c|}{ Xception } \\
\hline 6 & Média & Desvio & Média & Desvio \\
\hline 200 & $\mathbf{0 , 9 6 6 6 4}$ & 0,00430 & $\mathbf{0 , 9 9 9 5 6}$ & 0,00116 \\
\hline 300 & 0,96551 & 0,00391 & 0,98380 & 0,05091 \\
\hline 400 & 0,96175 & 0,00158 & 0,97766 & 0,07484 \\
\hline
\end{tabular}

\section{AgRadecimentos}

Os autores agradecem à CAPES (Coordenação de Aperfeiçoamento de Pessoal de Nível Superior), Ministério da Educação, código de financiamento 001.

\section{REFERÊNCIAS}

[1] R. Raghavendra, K. B. Raja, and C. Busch, "Presentation attack detection for face recognition using light field camera," IEEE Transactions on Image Processing, vol.24, n.3, pp.1060-1075, 2015.

[2] L. Li, J. Bao, T. Zhang, H. Yang, D. Chen, F. Wen, and B. Guo, "Face $\mathrm{x}$-ray for more general face forgery detection," IEEE/CVF Conference on Computer Vision and Pattern Recognition (CVPR), Seattle, USA, 14 a 19 de junho, pp.5000-5009, 2020.

[3] J. T. Jayan and R. P. Aneesh, "Image quality measures based face spoofing detection algorithm for online social media," International CET Conference on Control, Communication, and Computing (IC4), Thiruvanthapuram, India, 5 a 7 de julho, pp.245-249, 2018.

[4] A. Rattani, Z. Akhtar, and G. Foresti, "A preliminary study on identifying fabrication material from fake fingerprint images," IEEE Symposium Series on Computational Intelligence, Cape Town, África do Sul, 7 a 10 de dezembro, pp.362-366, 2015

[5] N. A. Kulkarni, and L. J. Sankpal, "Efficient approach determination for fake biometric Detection," International Conference on Computing, Communication, Control and Automation (ICCUBEA), Pune, India, 17 a 18 de agosto, pp.1-4, 2017.

[6] A. Singh, G. Jaswal, and A. Nigam, "FDSNet: Finger dorsal image spoof detection network using light field camera," IEEE 5th International Conference on Identity, Security, and Behavior Analysis (ISBA), Kobe, Japão, 13 a 14 de julho, pp.1-9, 2019.

[7] C. Zaghetto, M. Mendelson, A. Zaghetto, and F. de B. Vidal, "Liveness detection on touchless fingerprint devices using texture descriptors and artificial neural networks," IEEE International Joint Conference on Biometrics (IJCB), Denver, CO, USA, 1 a 4 de outubro, pp.406-412, 2017.

[8] A. Rattani, and A. Ross, "Minimizing the impact of spoof fabrication material on fingerprint liveness detector," IEEE International Conference on Image Processing (ICIP), Paris, França, 27 a 30 de outubro, pp.4992-4996, 2014.

[9] Khutlang, R., and Nelwamondo, F. V. (2014) Novelty detection-based internal fingerprint segmentation in Optical Coherence Tomography
Images. Second International Symposium on Computing and Networking, Shizuoka, Japão, 10 a 12 de dezembro, pp.556-559.

[10] F. Pala and B. Bhanu, "On the accuracy and robustness of deep triplet embedding for fingerprint liveness detection," IEEE International Conference on Image Processing (ICIP), Beijing, China, 17 a 20 de setembro, pp.116-120, 2017

[11] T. Chugh, and A. K. Jain, "Fingerprint spoof detector generalization," IEEE Transactions on Information Forensics and Security, vol.16, p.42-55, 2020.

[12] G. Wang, W. Kang, Q. Wu, Z. Wang, and J. Gao, "Generative adversarial network (GAN) based data augmentation for palmprint recognition," Digital Image Computing: Techniques and Applications (DICTA), Canberra, Australia, 10 a 13 de dezembro, pp.1-7, 2018.

[13] S. Tariq, S. Lee, H. Kim, Y. Shin, and S. S. Woo, "Detecting both machine and human created fake face images In the wild," 2nd International Workshop on Multimedia Privacy and Security - MPS '18, Toronto, Canadá, outubro, pp.81-87, 2018.

[14] T. Tan, X. Wang, Y. Fang, and W. Zhang, "The impact of data correlation on identification of computer-generated face images," 14 th Chinese Conference on Biometric Recognition, CCBR 2019, vol.11818, Zhuzhou, China, 12 a 13 de outubro, p.155-162, 2019.

[15] I. J. Goodfellow, J. Pouget-Abadie, M. Mirza, B. Xu, D. WardeFarley, S. Ozair, A. Courville, and Y. Bengio, "Generative adversarial networks," ArXiv:1406.2661 [Cs, Stat], 2014.

[16] P. Isola, J.-Y. Zhu, T. Zhou, and A. A. Efros, "Image-to-Image translation with conditional adversarial networks," IEEE Conference on Computer Vision and Pattern Recognition (CVPR), Honolulu, USA, 21 a 26 de julho, pp.5967-5976, 2017.

[17] F. Chollet, "Xception: Deep learning with depthwise separable convolutions," IEEE Conference on Computer Vision and Pattern Recognition (CVPR), Honolulu, 21-26 de julho, pp.1800-1807, 2017.

[18] O. Ronneberger, P. Fischer, and T. Brox, "U-Net: Convolutional networks for biomedical image segmentation," Medical Image Computing and Computer-Assisted Intervention - MICCAI, vol.9351, Munique, Alemanha, 5 a 9 de outubro. pp.234-241, 2015.

[19] A. Radford, L. Metz, and S. Chintala, "Unsupervised representation learning with deep convolutional generative adversarial networks," 4th International Conference on Learning Representations, (ICLR), San Juan, Porto Rico, 2 a 4 de maio, 2016.

[20] A. Krizhevsky, I. Sutskever, and G. E. Hinton, "ImageNet classification with deep convolutional neural networks," Communications of the ACM, vol.60, n.6, pp.84-90, 2012.

[21] S. Ioffe and C. Szegedy, "Batch normalization: Accelerating deep network training by reducing internal covariate shift," ICML'15: Proceedings of the 32nd International Conference on International Conference on Machine Learning, vol.37, Lille, França, 6 a 11 de julho, pp.448-456, 2015.

[22] C. Li and M. Wand, "Precomputed real-time texture synthesis with Markovian generative adversarial networks," Computer Vision (ECCV), 14th European Conference, vol. 9907, Amsterdã, Holanda, 8 a 16 de outubro, pp.702-716, 2016.

[23] K. He, X. Zhang, S. Ren, and J. Sun, "Deep residual learning for image recognition," ArXiv:1512.03385 [Cs], 2015.

[24] M. Cimpoi, S. Maji, I. Kokkinos, S. Mohamed, and A. Vedaldi, "Describing textures in the wild," IEEE Conference on Computer Vision and Pattern Recognition. Columbus, USA, 23-28 junho, 2014.

[25] K. Zuiderveld, "Contrast limited adaptive histogram equalization," In Graphics Gems: Vol. VIII.5 (Paul S. Hackbert eds.), Amsterdã, Elsevier, (pp. 474-485), 1994.

[26] S. M. Pizer, E. P. Amburn, J.D Austin, R. Cromartie, A. Geselowitz, T. Greer, and K. Zuiderveld, "Adaptive histogram equalization and Its variations," Computer Vision, Graphics, and Image Processing, vol.39, n.3, pp.355-368, 1987.

[27] R. C. Gonzalez and R. C. Woods, Processamento Digital de Imagens, (3rd ed.). Pearson Prentice Hall; pp. 46, 2010.

[28] O. M. Filho and H. V. Neto, Processamento Digital de Imagens, Brasport; pp. 27, 1999. 